\title{
Tác động của sự thích ứng tổ chức đến hiệu quả hoạt động của doanh nghiệp - Trường hợp các doanh nghiệp tại Thành phố Cần Tho
}

\section{The impact of organizational agility to the business performance - The case of enterprises in Can Tho City}

Nguyễn Thu Nha Trang ${ }^{1 *}$, Nguyễn Văn Hồng², Vũ Thị Minh Hiền ${ }^{3}$, Lưu Tiến Thuận ${ }^{4}$

${ }^{1}$ Trường Đại học Cần Thơ, Việt Nam

${ }^{2}$ Sở Kế hoạch Đầu tư Thành phố Cần Thơ, Việt Nam

${ }^{3}$ Kiểm toán Nhà nước KV5, Việt Nam

*Tác giả liên hệ, Email: ntntrang@ctu.edu.vn
THÔNG TIN

DOI: $10.46223 / \mathrm{HCMCOUJS.}$ soci.vi.15.1.602.2020

Ngày nhận: 13/03/2020

Ngày nhận lại: 15/04/2020

Duyệt đăng: 07/07/2020

Tù khóa:

doanh nghiệp, hiệu quả hoạt động, sự thích ứng tổ chức
TÓM TẮT 
Keywords:

enterprise, organizational agility, organization performance

on organization performance, in which the strongest impact is a decision-making factor compared with sensing and acting factors. The results also illustrate that medium and large scale enterprises have better organizational agility than small scale enterprises. Thereby, the paper gives some recommendations on improving organizational agility and organization performance as well.

\section{1. Đặt vấn đề}

Khi khoa học và công nghệ không ngừng thay đổi thì môi trường kinh doanh của các doanh nghiệp cũng bị ảnh hưởng không nhỏ và hậu quả là doanh nghiệp sẽ gặp khó khăn trong việc đạt được lợi thế cạnh tranh bền vững (D'Aveni, 1994). Mặt khác, trong bối cảnh nền kinh tế thị trường, khi nhu cầu và thị hiếu của khách hàng luôn biến đổi và bị tác động bởi nhiều yếu tố thì các doanh nghiệp không những buộc phải nâng cao sự linh hoạt thích ứng cho tổ chức, mà còn phải nâng cao việc chủ động trong việc dự đoán những thay đổi trước khi chúng làm ảnh hưởng đến hoạt động kinh doanh của doanh nghiệp. Điều đó cho thấy để tồn tại được trong môi trường có nhiều sự thay đổi thì các doanh nghiệp phải trở nên nhanh nhẹn hơn, thích nghi tốt hơn không những ở mọi khía cạnh của môi trường bên ngoài (chính trị, kinh tế, xã hội và công nghệ, ...) mà còn giải quyết tốt các vấn đề liên quan đến môi trường bên trong của doanh nghiệp (chiến lược nội bộ, cơ cấu tổ chức, ...) (Oosterhout, Waarts, \& Van Hillegersberg, 2006; Sharifi \& Zhang, 1999).

Tác giả Sambamurthy, Bharadwaj, và Grover (2003) cho rằng sự thích ứng tổ chức (organizational agility - OA) liên quan đến khả năng của doanh nghiệp hay tổ chức có thể phát hiện và nắm bắt các cơ hội cũng như giải quyết các mối đe dọa trong môi trường kinh doanh bất ổn. Mặt khác, Ganguly, Nilchiani, và Farr (2009), và Mathiassen và Pries-Heje (2006) nhấn mạnh đến sự thích ứng tổ chức được xem như một yếu tố kinh doanh chủ yếu và là yếu tổ thúc đẩy năng lực cạnh tranh cho tổ chức. Minh chứng cho ý kiến trên, trong nghiên cứu của Sull (2009) đã chỉ ra rằng 9 trên 10 giám đốc điều hành xếp hạng sự thích ứng tổ chức như một yếu tố then chốt quyết định sự thành công và phát triển trong kinh doanh cho tổ chức. Ngoài ra, tác giả Cascio (1989) cho rằng sự thích ứng tổ chức sẽ thúc đẩy khả năng cung ứng hàng hóa và dịch vụ chất lượng cao cho doanh nghiệp và đó là một yếu tố quan trọng đối với hiệu quả hoạt động kinh doanh của doanh nghiệp.

Lĩnh vực nghiên cứu liên quan đến sự thích ứng tổ chức nói chung cũng như sự tác động của nó đến hiệu quả hoạt động nói riêng đã được nhiều tác giả trên thế giới quan tâm và thực hiện. Tuy nhiên, vấn đề này lại khá mới mẻ đối với Việt Nam. Các tác giả thường nghiên cứu ở các doanh nghiệp hoạt động tại các thành phố lớn vì các doanh nghiệp này năng động và nhạy bén đối với sự thay đổi của môi trường kinh doanh. Mặc dù Thành phố Cần Thơ (TPCT) là trung tâm của vùng Đồng bằng sông Cửu Long với hệ thống kết cấu hạ tầng đã và đang được xây mới, nâng cấp và mở rộng mang tính chất liên kết vùng, quốc gia, tạo điều kiện cho các hoạt động đầu tư phát triển kinh tế - xã hội diễn ra thuận lợi hơn, nhưng các nghiên cứu ở các 
doanh nghiệp tại đây còn nhiều hạn chế. Vì vậy, việc nghiên cứu "Tác động của sụ thích úng tổ chức đến hiệu quả hoạt động của doanh nghiệp" ở TPCT là thật sự cần thiết. Trên cơ sở đó, bài viết đề xuất một số hàm ý quản trị nhằm nâng cao sự thích ứng tổ chức cũng như hiệu quả hoạt động cho các doanh nghiệp ở TPCT. Bài viết kỳ vọng đóng góp về mặt học thuật trong việc khai phá hướng nghiên cứu mới về sự thích ứng tổ chức trong trường hợp ở Việt Nam nói chung và TPCT nói riêng.

\section{Phương pháp luận và phương pháp nghiên cứu}

\subsection{Phương pháp luận}

\subsubsection{Khái niệm về sụ thích úng}

Từ đầu những năm 1990, mô hình liên quan đến sự thích ứng (agility) được xem là một trong những giải pháp cho môi trường quản trị năng động. Một tổ chức có sự thích ứng cao sẽ luôn trong tình thế sẵn sàng cho việc học hỏi những điều mới để gia tăng hiệu quả hoạt động từ việc nắm bắt các cơ hội mới. Qua lược khảo tài liệu, có nhiều khía cạnh và quan điểm khác nhau về sự thích ứng tổ chức, nên chưa có định nghĩa chung nào được chấp nhận trong giới học thuật hiện nay (Su, 2011). Năm 1991, viện Iacocca định nghĩa "sự thích ứng là một hệ thống sản xuất kết hợp với kỹ thuật công nghệ phần cứng và phần mềm, nguồn nhân lực và hệ thống thông tin nhằm đáp ứng nhu cầu ngày càng thay đổi của thị trường”. Theo March (1991), sự thích ứng bao gồm việc thăm dò và khai thác cơ hội để tạo ra lợi thế cạnh tranh cho mình. Sự khai thác là việc mở rộng và sàng lọc các năng lực, mô hình và công nghệ hiện có với kết quả tích cực, có thể dự đoán được. Trong khi đó, thăm dò là việc trải nghiệm các giải pháp thay thế với kết quả tiêu cực, không chắc chắn. Bên cạnh đó, nhóm tác giả Goldman, Nagel, và Preiss (1995) thì khẳng định sự thích ứng là phản ứng toàn diện với những thách thức như sự thay đổi không ngừng nghỉ của môi trường kinh doanh; của việc phân khúc thị trường liên tục; hay chất lượng sản phẩm cũng như hiệu quả hoạt động của doanh nghiệp được yêu cầu nâng cao theo yêu cầu của thị trường toàn cầu; hoặc hàng hóa và dịch vụ phải được định hình theo nhu cầu khách hàng.

\subsubsection{Khái niệm về sụ thích ứng tổ chức}

Sự thích ứng tổ chức là một khái niệm phức tạp và đa chiều. Một trong những khó khăn trong nghiên cứu là tìm ra được một khái niệm thống nhất cho sự thích ứng tổ chức. Lược khảo tài liệu cho thấy có nhiều khía cạnh đo lường cũng như mô hình khác nhau để đo lường sự thích ứng tổ chức. Tác giả Upton (1995) cho rằng sự thích ứng trong các tổ chức sản xuất là khả năng của một nhà máy trong việc thay đổi từ việc sản xuất một sản phẩm này sang một sản phẩm khác. Bên cạnh đó, các nghiên cứu của Richards (1996) hay Sanchez và Nagi (2001) lại cho rằng sự thích ứng tổ chức là một chiến lược tổng thế tập trung để phát triển tổ chức trong một môi trường không tiên đoán trước và sự phản ứng lại sự phức tạp của việc thay đổi liên tục. Sharifi và Zhang (2001) đề cập khái niệm về sự thích ứng tổ chức bao gồm hai yếu tố chính: thích nghi với những thay đổi (có thể dự báo và không dự báo được) và khai thác, tận dụng lợi thế của những thay đổi đó để tạo ra cơ hội cho mình. Tuy nhiên, nhóm tác giả Wadhwa và Rao (2003) thì tranh luận rằng sự thích ứng tổ chức tập trung nhiều hơn vào phản ứng sáng tạo, bởi vì nó giải quyết những thay đồi không lường trước được. Hay là khả năng phát hiện và nắm bắt các cơ hội của thị trường một cách nhanh chóng và bất ngờ (Sambamurthy et al., 2003) bởi vì nếu tổ chức có khả năng thích ứng thì sẽ liên tục cảm nhận được cơ hội đến từ môi trường kinh doanh. Từ đó họ sẽ tận dụng các tri thức và tài sản cần thiết để nắm bắt những cơ hội đó. 
Sự thích ứng tổ chức là việc thực hiện thành công các nguyên tắc cạnh tranh như tốc độ, linh hoạt, đổi mới sáng tạo và chất lượng bằng cách kết hợp các nguồn lực và thực hiện tốt nhất để cung cấp sản phẩm và dịch vụ theo định hướng khách hàng trong môi trường với nhiều thay đổi. Sự hài lòng của khách hàng cũng như nhân viên là một trong những mục tiêu của sự thích ứng của tổ chức. Sự thích ứng trong tổ chức phải thực hiện được cách tổ chức, quy trình thực hiện và tạo ra sản phẩm theo cách phản ứng một cách hợp lý với những thay đổi trong một khoảng thời gian thích hợp (Balaji et al., 2014; Pavlou \& Sawy, 2010). Đây cũng là khái niệm mà nghiên cứu dựa vào để thực hiện cho việc xây dựng thang đo của sự thích ứng tổ chức.

\subsubsection{Khái niệm về hiệu quả hoạt động}

Một trong các câu hỏi quan trọng mà các nhà quản lý doanh nghiệp đang băn khoăn là tại sao một số doanh nghiệp lại thành công, trong khi số khác lại thất bại. Hiệu quả hoạt động của doanh nghiệp trở thành vấn đề quan trọng nhất cho mọi tổ chức hoạt động vì lợi nhuận cũng như không lợi nhuận. Việc nhà quản lý cần biết nhân tố nào tác động hiệu quả hoạt động của doanh nghiệp là rất quan trọng, bởi vì điều này giúp họ thực hiện những bước đi thích hợp từ đó giúp họ đạt được mục tiêu do tổ chức đề ra. Thuật ngữ hiệu quả (performance) đôi khi bị nhầm lẫn với năng suất "productivity". Theo tác giả Lagos (2001) năng suất thể hiện khối lượng công việc hoàn thành trong một khoảng thời gian nhất đinh; về cơ bản, năng suất được đo bằng cách so sánh lượng sản phẩm/dịch vụ được thực hiện so với lượng đầu vào được sử dụng trong sản xuất. Trong khi đó hiệu quả là một chỉ số rộng hơn, có thể bao gồm năng suất cũng như chất lượng, tính nhất quán và những yếu tố khác. Tuy nhiên, việc xác định, khái niệm hóa cũng như đo lường hiệu quả hoạt động là nhiệm vụ không đơn giản.

Nhiều nhà nghiên cứu đã đưa ra nhiều ý kiến và định nghĩa khác nhau về hiệu quả hoạt động, và điều này vẫn còn đang là vấn đề đang tranh cãi giữa các nhà nghiên cứu về doanh nghiệp/tổ chức (Barney, 1997). Tác giả Daft (2000) cho rằng hiệu quả hoạt động của doanh nghiệp là năng lực của doanh nghiệp đạt được các mục tiêu đã đề ra bằng cách sử dụng các nguồn lực một cách có kết quả và hiệu quả. Đồng quan điểm với Daft (2000), tác giả Lagos (2001) đã định nghĩa hiệu quả hoạt động của tổ chức như khả năng của tổ chức để đạt được mục đích và mục tiêu của tổ chức. Riêng Gimeno (2002) xem hiệu quả tương đương với 3Es phổ biến: kinh tế (economy), kết quả (effectiveness) và hiệu quả (efficiency) của một chương trình hoặc hoạt động cụ thể. Ngoài ra, hiệu quả hoạt động của tổ chức phản ánh khả năng của một tổ chức thích ứng được các yêu cầu của các bên liên quan và có thể tồn tại trên thương trường (Griffin, 2003). Hiệu quả hoạt động của tổ chức còn được biết đến như là kết quả của các hoạt động được thực hiện bởi các thành viên của tổ chức để đo lường mức độ hoàn thành các mục tiêu của nó (Chung và Lo, 2007; Ho, 2008). Vậy, hiệu quả hoạt động của tổ chức không chỉ mâu thuẫn về vấn đề định nghĩa, mà còn mâu thuẫn về vấn đề khái niệm hóa (Heffernan \& Flood, 2000).

Đối với việc đo lường hiệu quả hoạt động, hiện tại có hai khung đo lường chủ yếu, đó là: "thẻ điểm cân bằng" (balanced scored card) của Kaplan và Norton (1996); và "các mức độ thành công" (success dimensions) của Shenhar và Dvir's (1996). Tuy nhiên, hai khung đo lường này lại có nhược điểm là không đề cập đến khía cạnh nguồn nhân lực của tổ chức. Chính vì thế, Maltz Shenhar, và Reilly (2003) đã đề xuất một khía cạnh mới trong việc đề cập đến hiệu quả hoạt động đó là vấn đề phát triển nhân sự trong tổ chức. Đây cũng là cơ sở để xây dựng thang đo hiệu quả hoạt động cho nghiên cứu này. 


\subsection{Phương pháp nghiên cúu}

\subsubsection{Mồ hìh nghiên cúu}

Mục tiêu của nghiên cứu nhằm xác định các doanh nghiệp cảm nhận như thế nào đối với sự thay đổi, từ đó có những quyết định liên quan nào và cuối cùng là hành động cụ thể là gì đối với sự thay đổi đó và cuối cùng là tác động như thế nào đến hiệu quả hoạt động của doanh nghiệp. Cho nên, thang đo của sự thích ứng bao gồm 3 yếu tố cơ bản (Bảng 1): sự cảm nhận, ra quyết định và hành động (Pavlou \& Sawy, 2010), được phát triển thành 15 mục hỏi.

\section{Bảng 1}

Các thành phần của sự thích ứng

\begin{tabular}{lll}
\hline \multicolumn{1}{c}{ Thành phần } & \multicolumn{1}{c}{ Diễn giải } & Tác giả \\
\hline Khả năng cảm nhận & $\begin{array}{l}\text { Phát hiện và nắm bắt các sự kiện kinh doanh quan trọng một } \\
\text { cách kịp thời }\end{array}$ & $\begin{array}{l}\text { Pavlou \& } \\
\text { Sawy }\end{array}$ \\
Ra quyết định & $\begin{array}{l}\text { Phân tích các sự kiện, xác định các cơ hội và thách thức nhằm } \\
\text { đưa ra các hành động thích hợp kịp thời }\end{array}$ & $\begin{array}{l}(2010) \text {; Park } \\
(2011) ;\end{array}$ \\
Hành động & $\begin{array}{l}\text { Linh hoạt thiết lập lại các nguồn lực của tổ chức, hay có thể } \\
\text { thay đổi quy trình kinh doanh hay giới thiệu các cải tiến mới } \\
\text { cho thị trường một cách kịp thời }\end{array}$ & \\
& & \\
\hline
\end{tabular}

Nguồn: Tổng hợp của tác giả (2019)

Hiệu quả hoạt động trong nghiên cứu này đề cập đến (1) hiệu quả tài chính, (2) quy trình nội bộ, và (3) phát triển nhân sự. Như vậy, nghiên cứu này đề cập đến chỉ số hiệu quả năng động đa chiều được đề xuất bởi Maltz và cộng sự (2003) và được phát triển thành 11 mục hỏi để đo lường hiệu quả hoạt động.

Dựa trên cơ sở tổng kết lý thuyết và các nghiên cứu trước đây về sự thích ứng tổ chức, tác giả đề xuất mô hình nghiên cứu thể hiện ở Hình 1 .

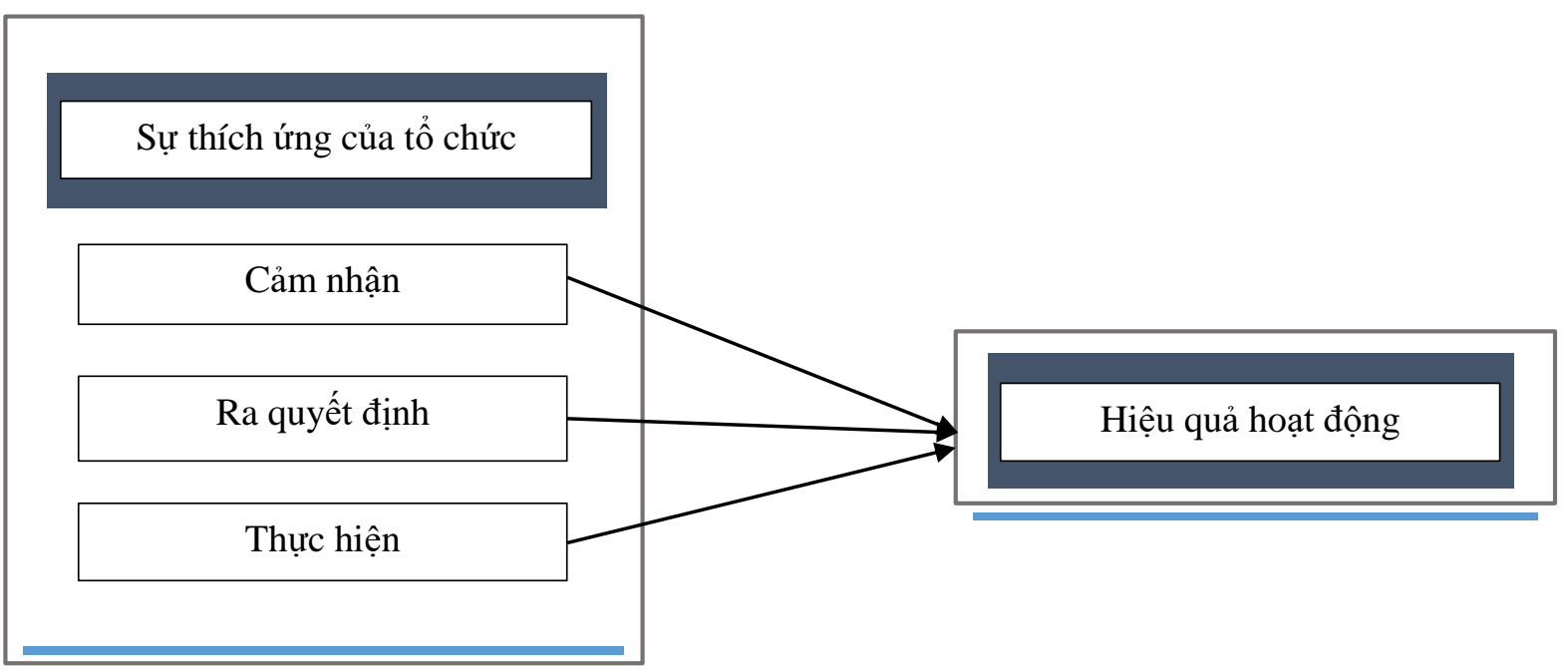

Hình 1. Mô hình nghiên cứu đề xuất 


\section{Giả thuyết nghiên cứu:}

Sự thích ứng của tổ chức có mối quan hệ tích cực đến hiệu quả hoạt động của tổ chức vì sự thích ứng có thể giúp doanh nghiệp có khả năng đối phó với sự thay đổi của môi trường, giúp doanh nghiệp phát triển cũng như cung cấp được những sản phẩm và dịch vụ có chất lượng cao (Alegre \& Sard, 2015; Shahrabi, 2012). Cho nên, các tác giả trước đây đã nhận định rằng sự thích ứng của tổ chức có mối quan hệ tích cực đến hiệu quả hoạt động (Tallon \& Pinsonneault, 2011). Vì vậy, giả thuyết sau được xây dựng:

\section{$H_{1}$ : Sự thich ứng tổ chức có tác động tích cực đến hiệu quả hoạt động của doanh nghiệp}

\section{Giả thuyết phụ:}

$H_{1-1}$ : Việc nâng cao cảm nhận có tác động tích cực đến hiệu quả hoạt động của doanh nghiệp

$H_{1-2}$ : Nâng cao việc ra quyết định có tác động tích cục đến hiệu quả hoạt động của doanh nghiệp $H_{1-3}$ : Việc nâng cao hành động có tác động tích cực đến hiệu quả hoạt động của doanh nghiệp

\subsubsection{Phưong pháp thu thập dĩu liệu}

Về xác định cỡ mẫu: theo tác giả Hair, Black, Babin, Anderson, và Tatham (2006) để sử dụng phân tích nhân tố EFA, kích thước mẫu tốt hơn là 100 và tỉ lệ quan sát/biến đo lường từ $5: 1$. Tác giả Hoang và Chu (2008) cũng cho rằng cỡ mẫu ít nhất phải bằng 4-5 lần số biến. Bên cạnh đó, nghiên cứu có sử dụng phương pháp phân tích SEM, đòi hỏi cỡ mẫu đủ lớn vì nó dựa vào lý thuyết phân phối mẫu (Raykov \& Widaman, 1995). Theo Hoyle (1995) để có độ tin cậy trong kiểm định độ thích hợp của mô hình SEM, kích thước mẫu từ trên 200 là đạt yêu cầu. Với 15 biến đo lường của thang đo sự thích ứng, và 11 biến đo lường của thang đo hiệu quả hoạt động, để tiến hành phân tích EFA, cỡ mẫu tối thiểu phải là 26 x $4=104$ quan sát. Để đảm bảo thỏa mãn cỡ mẫu theo đề xuất của Hoang và Chu (2008), và đảm bảo kích thước mẫu tối thiểu cho phù hợp với các phương pháp phân tích, bài nghiên cứu xác định kích thước mẫu lớn hơn 200 . Do đó, nghiên cứu tiến hành khảo sát 250 quan sát bằng phương pháp chọn mẫu phi xác suất và theo cách chọn mẫu hạn ngạch (quota). Đối tượng phỏng vấn trực tiếp là nhân viên và nhà quản lý của các doanh nghiệp tại TPCT. Kết quả có 249 phiếu khảo sát đạt yêu cầu và được dùng để phân tích.

\subsubsection{Phương pháp phân tích số liệu}

Mô hình nghiên cứu được kiểm định thông qua các bước sau: (1) Kiểm định độ tin cậy của thang đo Cronbach's Alpha; (2) Phân tích nhân tố khám phá (EFA) và phân tích nhân tố khẳng định (CFA) để kiểm định sự phù hợp của mô hình với dữ liệu thị trường; (3) Phân tích mô hình cấu trúc tuyến tính (SEM) để đo lường sự tác động của sự thích ứng tổ chức đến hiệu quả hoạt động của doanh nghiệp; và (4) phân tích cấu trúc đa nhóm theo quy mô doanh nghiệp.

\section{Kết quả và thảo luận}

\subsection{Mô tả đặc điểm mẫu khảo sát}

Dựa vào số lượng doanh nghiệp ở các quận của TPCT, số quan sát được thu thập tương ứng ở các quận như sau: quận Ninh Kiều (100); Cái Răng (65); Bình Thủy (57); Ô môn (13); và quận Thốt nốt (14). Hơn $61 \%$ đáp viên tham gia khảo sát giữ chức vụ quản lý (từ giám đốc đến phó trưởng phòng) và số còn lại là nhân viên bán hàng, marketing, giao dịch, v.v. Trong nghiên cứu này, tỉ lệ nam được khảo sát chiếm hơn $58 \%$. Về quy mô, có $68,3 \%$ là doanh nghiệp nhỏ, 31,7\% là doanh nghiệp vừa và lớn. 


\section{Bảng 2}

Đặc điểm mẫu khảo sát

\begin{tabular}{|l|l|c|c|}
\hline \multicolumn{1}{|c|}{ Thành phần } & \multicolumn{1}{|c|}{ Đặc tính } & Tần số & Tỉ lệ (\%) \\
\hline \multirow{3}{*}{ Giới tính } & Nữ & 103 & 41,4 \\
& Nam & 146 & 58,6 \\
\hline \multirow{3}{*}{ Vị trí công việc } & Giám đốc & 70 & 28,1 \\
& Phó giám đốc \\
& Trưởng, phó phòng & 30 & 12,0 \\
& Nhân viên & 72 & 21,7 \\
& & 95 & 38,2 \\
\hline \multirow{3}{*}{ Quy mô DN } & Nhỏ & 170 & 68,3 \\
& Lớn và vừa & 79 & 31,7 \\
\hline & TỔNG & 249 & 100,0 \\
\hline
\end{tabular}

Nguồn: Kết quả khảo sát 249 doanh nghiệp (2019)

\section{2. Đánh giá độ tin cậy của thang đo}

Kiểm định độ tin cậy thang đo Cronbach's Alpha được sử dụng để loại bỏ các biến không phù hợp hay các biến rác trong quá trình nghiên cứu, trước khi tiến hành phân tích nhân tố. Tiêu chuẩn để chọn thang đo là có độ tin cậy Cronbach's Alpha từ 0,6 trở lên nhưng tốt nhất là lớn hơn 0,7 (Nunnally \& Berstein, 1994). Kết quả phân tích ở Bảng 3 cho thấy hệ số Cronbach's Alpha của thang đo đều lớn hơn 0,7 và hệ số tương quan biến tổng của các biến quan sát đều lớn hơn 0,5 . Điều này cho thấy các biến đo lường là tốt (Nunnally \& Berstein, 1994) và có thể sử dụng cho phân tích nhân tố khám phá (EFA).

\subsection{Phân tích nhân tố khám phá (EFA)}

Bài viết tiến hành phân tích EFA với 25 biến sau khi thỏa yêu cầu về kiểm định Cronbach's Alpha của từng thang đo. Trong quá trình phân tích EFA, các biến có hệ số tải nhân tố bé hơn 0,5 lần lượt được loại ra để đảm bảo có ý nghĩa thực tiễn cao (Hair et al., 2006). Phương pháp trích hệ số sử dụng là phương pháp thành phần chính (Principal axis factoring) với phép xoay cho phương sai tối đa (prorimax) và điểm dừng khi các yếu tố có phương sai tổng hợp của từng nhân tố (eigenvalue $\geq 1$ ). 


\section{Bảng 3}

Kết quả kiểm định Cronbach's Alpha và phân tích EFA của từng thang đo

\begin{tabular}{|c|c|c|c|c|c|}
\hline Biến & $\begin{array}{c}\text { Hệ số } \\
\text { tải } \\
\text { nhân tố }\end{array}$ & $\begin{array}{l}\text { Giá trị } \\
\text { Eigen }\end{array}$ & $\begin{array}{c}\text { Tổng } \\
\text { phương sai } \\
\text { trích (\%) }\end{array}$ & $\begin{array}{c}\text { Kiểm } \\
\text { định } \\
\text { KMO }\end{array}$ & $\begin{array}{c}\text { Hệ số } \\
\text { Cronbach's } \\
\text { Alpha }\end{array}$ \\
\hline Sự thích ứng & & & 64,6 & $\mathbf{0 , 7 9 2}$ & \\
\hline - Cảm nhận & & 1,229 & & & 0,777 \\
\hline OA_S1 & 0,647 & & & & \\
\hline OA_S2 & 0,761 & & & & \\
\hline OA_S3 & 0,812 & & & & \\
\hline - Ra quyết định & & 4,769 & & & 0,907 \\
\hline OA_DM2 & 0,916 & & & & \\
\hline OA_DM3 & 0,767 & & & & \\
\hline OA_DM4 & 0,938 & & & & \\
\hline OA_DM5 & 0,752 & & & & \\
\hline - Hành động & & 1,510 & & & 0,784 \\
\hline OA_A1 & 0,688 & & & & \\
\hline OA_A5 & 0,947 & & & & \\
\hline OA_A6 & 0,612 & & & & \\
\hline Hiệu quả hoạt động & & & 65,9 & $\mathbf{0 , 8 3 1}$ & \\
\hline - Hiệu quả tài chính & & 1,097 & & & 0,771 \\
\hline BP_FP1 & 0,598 & & & & \\
\hline BP_FP2 & 0,777 & & & & \\
\hline BP_FP3 & 0,761 & & & & \\
\hline Quy trình nội bộ & & 4,482 & & & 0,907 \\
\hline BP_IP1 & 0,911 & & & & \\
\hline BP_IP2 & 0,887 & & & & \\
\hline BP_IP3 & 0,803 & & & & \\
\hline - Phát triển nhân sự & & 1,365 & & & 0,843 \\
\hline BP_HD1 & 0,736 & & & & \\
\hline BP_HD2 & 0,839 & & & & \\
\hline BP_HD3 & 0,793 & & & & \\
\hline
\end{tabular}

Nguồn: Kết quả khảo sát 249 doanh nghiệp (2019)

Kết quả biến OA_A2, OA_3, OA_4, OA_A7, IP4 và HD4 bị loại khỏi mô hình phân tích vì hệ số tải < 0,5. Kết quả phân tích KMOOA $=0,792 ; \mathrm{KMOBP}=0,831(0,5 \mathrm{KMO} 1)$ với tổng phương sai trích đều lớn hơn $50 \%$ ) và kiểm định Bartlett's về tương quan của các biến có giá trị Sig $=0,000<0,05$ (Bảng 3) cho thấy các biến có tương quan với nhau (Hoang \& Chu, 2008) và dữ liệu phù hợp để thực hiện phân tích nhân tố CFA. Kết quả cho thấy, có 6 nhóm nhân tố được hình thành và không có sự xáo trộn nên các nhân tố này sẽ không thay đổi tên nhóm. 


\subsection{Phân tích nhân tố khẳng định (CFA) các thang đo}

Kiểm định CFA bao gồm bốn kiểm định. (1) Kiểm định mức độ phù hợp chung của mô hình qua việc kiểm định các chỉ tiêu: cảm nhận $(\mathrm{S})$, ra quyết định $(\mathrm{OA} \mathrm{DM})$, hành động (OA_A), hiệu quả tài chính (BP_FP), quy trình nội bộ (BP_IP), và chính sách nhân sự (BP_HD).

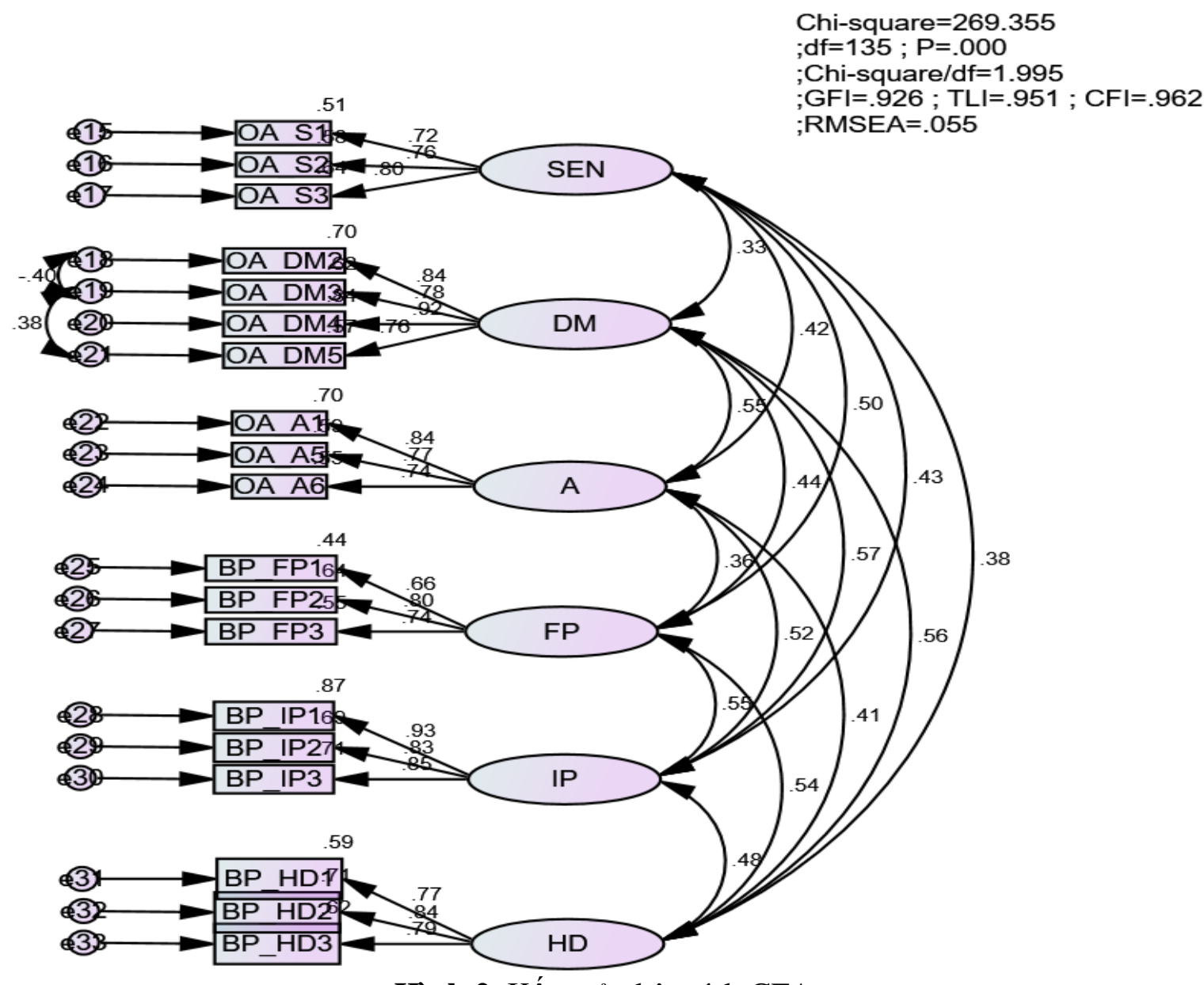

Hình 2. Kết quả phân tích CFA

Hình 2 trình bày kết quả phân tích nhân tố khẳng định (CFA) các thang đo. Cụ thể kiểm định Chi-bình phương của mô hình tới hạn có giá trị p-value $=0,000<0,05$, các chỉ tiêu Chisquare/df $=1,995(<2)$, các giá trị $\mathrm{TLI}=0,951$ và $\mathrm{CFI}=0,962$ đều lớn hơn 0,9 và $\mathrm{RMSEA}=$ $0,055<0,08$. Như vậy, các chỉ tiêu đều đạt yêu cầu nên mô hình phù hợp với dữ liệu thị trường (Nguyen \& Nguyen, 2008). Các trọng số chưa chuẩn hóa đều có ý nghĩa thống kê (p-value < $0,05)$ và các trọng số đã chuẩn hóa đều $>0,5$ nên thang đo đạt được giá trị hội tụ (Gerbing \& Anderson, 1988). Bên cạnh đó, kết quả kiểm định cho thấy các giá trị p-value đều <0,05 nên hệ số tương quan của từng cặp khái niệm < 1 ở độ tin cậy 95\%. Do đó, các khái niệm đạt giá trị phân biệt. (2) Kiểm định độ tin cậy của thang đo thông qua: Hệ số tin cậy tổng hợp (Composite Reliability $-\mathrm{CR}) \geq 0,7$ và Phương sai trích trung bình (Average Variance Extracted - AVE) phải có giá trị tối thiểu là $50 \%$ (Hair et al., 2006). Kết quả Bảng 4 cho thấy $\mathrm{CR}$ dao động từ 0,807 đến 0.915 , đều thỏa yêu cầu $\geq 0,7$ và $A V E$ dao động từ 52,8 đến $75,3 \%$, thỏa yêu cầu $\geq$ $50 \%$. Điều này cho thấy thang đo đảm bảo độ tin cậy. (3) Kiểm định giá trị hội tụ qua việc xem 
xét các hệ số tải đã chuẩn hóa là $0,641(\geq 0,5)$ nên thang đo đảm bảo tốt giá trị hội tụ. (4) Kiểm định giá trị phân biệt: Điều kiện để thang đo đạt giá trị phân biệt là hệ số tương quan giữa các yếu tố phải khác biệt so với 1 (D. K. Nguyen, 2009, p. 47). Qua bảng tính excel, các giá trị p_value đều nhỏ hơn 0,05 nên hệ số tương quan từng cặp khái niệm có khác biệt so với 1 ở độ tin cậy $95 \%$ và cụ thể hơn là < 1 . Do đó các khái niệm đạt được độ giá trị phân biệt.

\section{Bảng 4}

Tóm tắt kết quả kiểm định thang đo

\begin{tabular}{|l|c|c|c|c|c|c|c|c|c|c|}
\hline & CR & AVE & MSV & MaxR(H) & $\begin{array}{c}\text { Quy } \\
\text { trình } \\
\text { nội } \\
\text { bộ }\end{array}$ & $\begin{array}{c}\text { Cảm } \\
\text { nhận }\end{array}$ & $\begin{array}{c}\text { Ra } \\
\text { quyết } \\
\text { định }\end{array}$ & $\begin{array}{c}\text { Hành } \\
\text { dộng }\end{array}$ & $\begin{array}{c}\text { Hiệu } \\
\text { quả tài } \\
\text { chính }\end{array}$ & $\begin{array}{c}\text { Phát } \\
\text { triển } \\
\text { nhân } \\
\text { sự }\end{array}$ \\
\hline $\begin{array}{l}\text { Quy trình nội } \\
\text { bộ }\end{array}$ & 0,901 & 0,753 & 0,306 & 0,920 & $\mathbf{0 , 8 6 8}$ & & & & & \\
\hline Cảm nhận & 0,807 & 0,583 & 0,158 & 0,813 & 0,359 & $\mathbf{0 , 7 6 4}$ & & & & \\
\hline Ra quyêt định & 0,915 & 0,728 & 0,310 & 0,917 & 0,553 & 0,291 & $\mathbf{0 , 8 5 3}$ & & & \\
\hline Hành động & 0,814 & 0,594 & 0,310 & 0,828 & 0,505 & 0,361 & 0,557 & $\mathbf{0 , 7 7 1}$ & & \\
\hline $\begin{array}{l}\text { Hiệu quả tài } \\
\text { chính }\end{array}$ & 0,769 & 0,528 & 0,288 & 0,784 & 0,535 & 0,397 & 0,405 & 0,334 & $\mathbf{0 , 7 2 6}$ & \\
\hline $\begin{array}{l}\text { Phát triển } \\
\text { nhân sự }\end{array}$ & 0,834 & 0,626 & 0,288 & 0,841 & 0,484 & 0,307 & 0,494 & 0,381 & 0,537 & $\mathbf{0 , 7 9 1}$ \\
\hline
\end{tabular}

Nguồn: Kết quả khảo sát 249 doanh nghiệp (2019)

\subsection{Phân tích tác động của sụ̂ thích ứng tổ chức đến hiệu quả họ̣t động của doanh nghiệp tại TPCT}

Kiểm định mô hình lý thuyết SEM

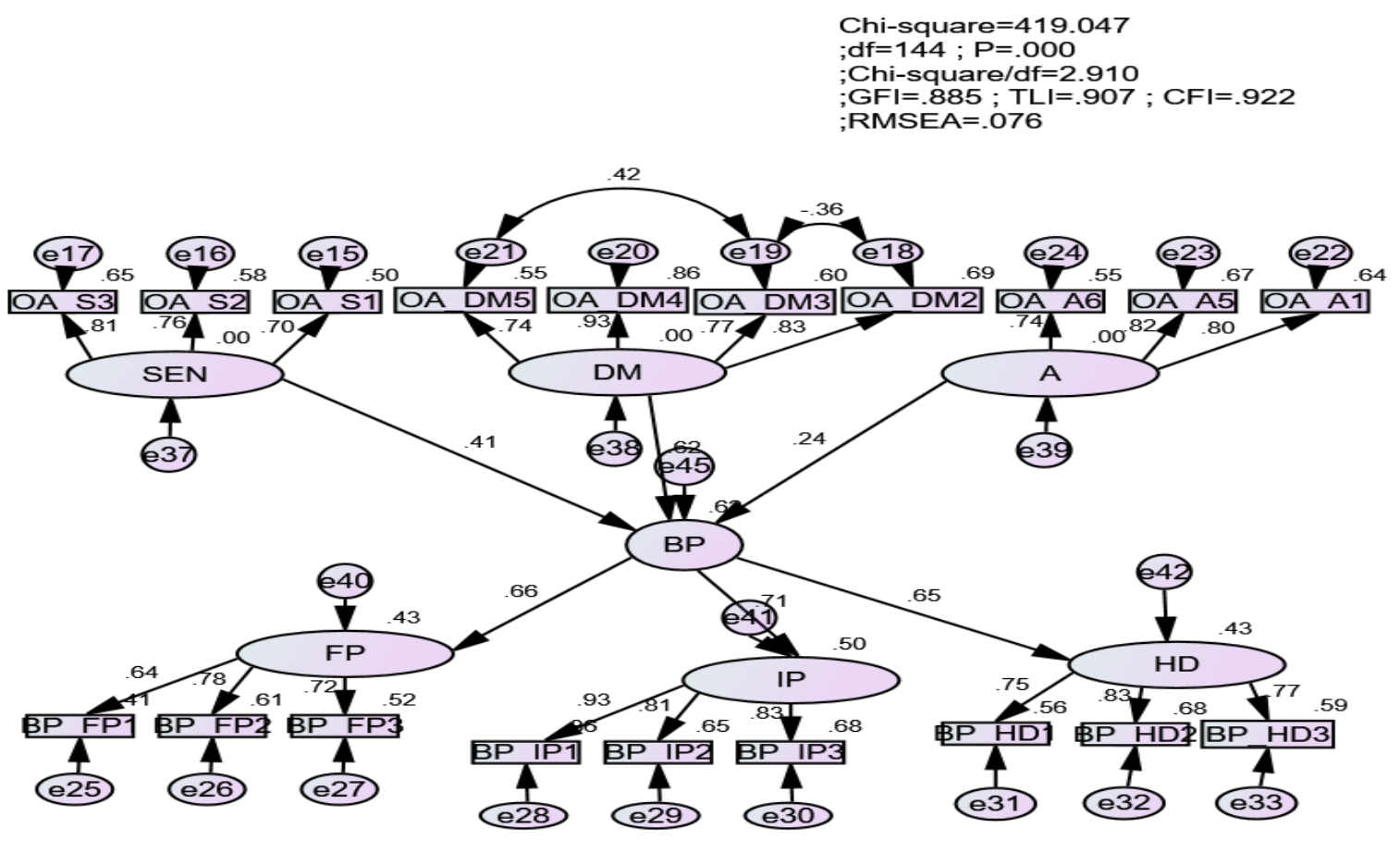

Hình 3. Mô hình SEM đã chuẩn hóa 
Kết quả ước lượng của mô hình lý thuyết được trình bày ở Hình 3. Mô hình này có 1 khái niệm phụ thuộc và 3 khái niệm độc lập (cảm nhận, ra quyết định và hành động). Mô hình lý thuyết có 144 bậc tự do; các giá trị chi-square $=419,047 ; \mathrm{p}$-value=0,000; Chi-square/df $=$ $2,910(<3)$; các hệ số TLI và CFI đều > 0,9; RMSEA =0,076 $(<0,8)$ chỉ ra mô hình nghiên cứu phù hợp với dữ liệu thị trường. Kết quả ước lượng chuẩn hóa của các tham số chính cho thấy các mối quan hệ giả thuyết đều có ý nghĩa thống kê (p-value<0,05): Sự cảm nhận và hiệu quả hoạt động $(0,000)$, ra quyết định và hiệu quả hoạt động $(0,000)$ và hành động với hiệu quả hoạt động $(0,000)$. Hơn nữa, các trọng số chuẩn hóa đều mang dấu dương cho thấy các nhân tố cảm nhận, ra quyết định, và hành động có tác động thuận chiều đến hiệu quả hoạt động của doanh nghiệp, với mức ảnh hưởng lần lượt là 0,$418 ; 0,620$ và 0,250 . Khả năng giải thích cho các biến phụ thuộc trong mô hình cũng khá cao, cụ thể hiệu quả hoạt động của doanh nghiệp được giải thích $81,0 \%$ bởi sự thích ứng tổ chức. Do đó, giả thuyết $\mathrm{H} 1$ được chấp nhận, nghĩa là sự thích ứng tổ chức có tác động tích cực đến hiệu quả hoạt động của doanh nghiệp.

Bảng 5 thể hiện việc ra quyết định (DM) có tác động mạnh nhất đến hiệu quả hoạt động của doanh nghiệp $(\mathrm{BP})$, kế tiếp là sự cảm nhận $(\mathrm{SEN})$, và cuối cùng là hành động $(\mathrm{A})$, với các chỉ số ước lượng cụ thể lần lượt tương ứng là 0,$624 ; 0,413$; và 0,238 . Qua đó, có thể thấy sự cảm nhận, ra quyết định và hành động là những nhân tố mà nếu mất đi sẽ làm ảnh hưởng đến hiệu quả hoạt động của doanh nghiệp. Kết quả nghiên cứu cũng tương đồng với kết quả các nghiên cứu của Abolfazl và Mehrdad (2016); Anas (2016); Najmi và cộng sự (2016); và Wageeh (2016).

\section{Bảng 5}

Các trọng số chưa chuẩn hóa và đã chuẩn hóa trong kiểm định mô hình SEM

\begin{tabular}{|lc|cccc|c|}
\hline & \multicolumn{4}{|c|}{ Chưa chuẩn hóa } & Đã chuẩn hóa \\
\cline { 3 - 7 } & & Ước lưọng & S,E, & C,R, & P & Ước lưọng \\
\hline BP <--- & SEN & 0,178 & 0,034 & 5,269 & $* * *$ & 0,413 \\
BP <--- & DM & 0,298 & 0,044 & 6,792 & $* * *$ & 0,624 \\
BP <--- & A & 0,120 & 0,033 & 3,595 & $* * *$ & 0,238 \\
\hline
\end{tabular}

Nguồn: Kết quả khảo sát 249 doanh nghiệp (2019)

Sự cảm nhận có ý nghĩa và tác động tích cực đến hiệu quả hoạt động kinh doanh của doanh nghiệp. Nếu doanh nghiệp không có khả năng cảm nhận được những thay đổi xung quanh hoạt động kinh doanh của mình thì doanh nghiệp sẽ khó tồn tại. Bên cạnh đó, việc ra quyết định liên quan đến việc cải thiện trong nội bộ doanh nghiệp một cách phù hợp sẽ giúp doanh nghiệp gia tăng khả năng thích ứng của mình với sự thay đổi của môi trường hoạt động kinh doanh bên ngoài. Đây cũng là yếu tố tác động mạnh nhất đến biến phụ thuộc của mô hình nghiên cứu. Nhân tố cuối cùng trong sự thích ứng tổ chức là hành động, góp phần tác động không nhỏ đến hiệu quả hoạt động của doanh nghiệp. Nhân tố này giúp doanh nghiệp nhanh chóng phát triển cơ hội hay tối giảm những thách thức khi những thay đổi xảy ra. 


\subsection{Kiểm định sụ khác biệt về tác động của sụ thích ứng tổ chức đến hiệu quả hoạt động đối với các doanh nghiệp có quy mô khác nhau tại Thành phố Cần Tho}

Phương pháp phân tích cấu trúc đa nhóm được sử dụng để so sánh mô hình lý thuyết theo quy mô của 2 nhóm doanh nghiệp là doanh nghiệp nhỏ, và doanh nghiệp vừa và lớn. Phương pháp phân tích đa nhóm sử dụng trong nghiên cứu này bao gồm khả biển và bất biến từng phần. Trong mô hình khả biến, các tham số ước lượng trong từng mô hình của các nhóm không bị ràng buộc (Hình 4). Trong mô hình bất biến từng phần, thành phần đo lường (biến quan sát) không bị ràng buộc nhưng các mối quan hệ giữa các khái niệm trong mô hình bị ràng buộc có giá trị như nhau cho cả hai nhóm.

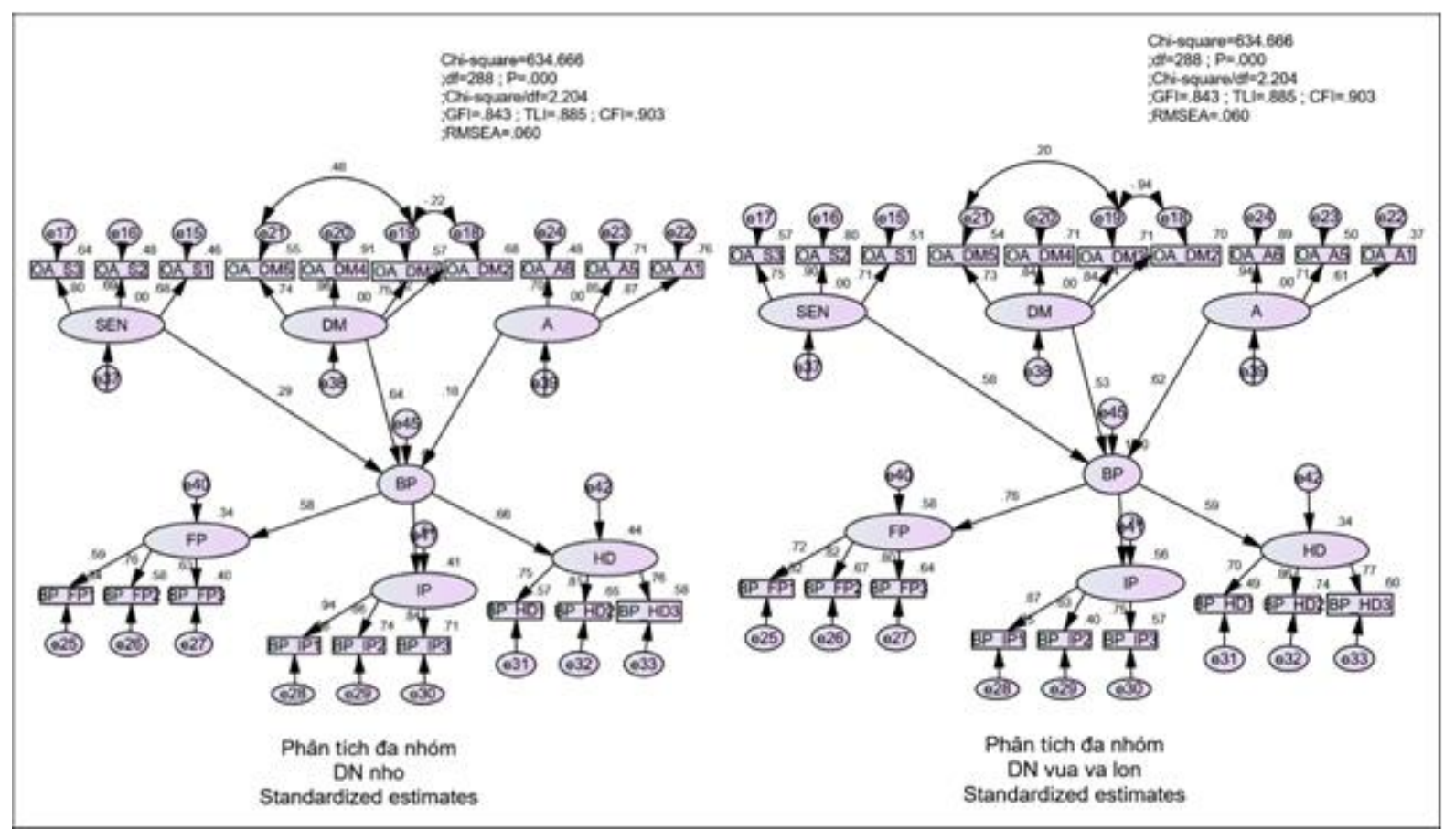

Hình 4. Mô hình SEM khả biến theo quy mô doanh nghiệp

Kiểm định khác biệt Chi-bình phương được dùng để so sánh hai mô hình (bất biến và khả biến từng phần). Nếu kiểm định khác biệt cho thấy mức khác biệt của hai mô hình không có ý nghĩa thì mô hình bất biến được chọn (bậc tự do cao hơn). Ngược lại, nếu sự khác biệt Chibình phương có ý nghĩa $(<5 \%)$ thì sẽ chọn mô hình khả biến (có độ tương thích cao hơn). Kết quả Bảng 6 cho thấy kiểm định Chi-square có sự khác biệt về tác động của sự thích ứng tổ chức đến hiệu quả hoạt động của doanh nghiệp giữa 2 quy mô doanh nghiệp nhỏ; và doanh nghiệp vừa và lớn. Do $\mathrm{p}$-value $=0,000<0,05$ nên mô hình khả biến sẽ được chọn:

\section{Bảng 6}

Kết quả kiểm định Chi-square

\begin{tabular}{|l|c|c|c|}
\hline & Chi-square & Df & \multirow{2}{*}{ P-value } \\
\hline Mô hình khả biến & 634,686 & 288 & \multirow{2}{*}{0,000} \\
\hline Mô hình bất biến & 665,539 & 291 & \\
\hline Sai biệt & 30,853 & 3 & \\
\hline
\end{tabular}

Nguồn: Kết quả khảo sát 249 doanh nghiệp (2019) 
Kết quả Bảng 7 cho thấy nhìn chung các thành phần của sự thích ứng tác động đến hiệu quả hoạt động của doanh nghiệp nhỏ thấp hơn so với các doanh nghiệp vừa và lớn. Cảm nhận của doanh nghiệp vừa và lớn có tác động mạnh đến hiệu quả hoạt động khi so sánh với doanh nghiệp nhỏ. Điều này có được là do doanh nghiệp lớn có nguồn lực thích hợp để phân tích vả cảm nhận được thị trường tốt hơn các doanh nghiệp nhỏ và vừa. Tuy nhiên, doanh nghiệp vừa và lớn có sự tác động của việc ra quyết định đến hiệu quả hoạt động thấp hơn so với loại doanh nghiệp nhỏ. Sự khác biệt này không lớn lắm. Điều này có thể do các doanh nghiệp lớn ở TPCT, chủ yếu là chi nhánh trong một tập đoàn doanh nghiệp lớn (Vingroup, các công ty bảo hiểm nhân thọ, các ngân hàng thương mại...), chịu sự chi phối không nhỏ từ các công ty mẹ, từ hội sở chính nên việc ra quyết định không được nhanh như các doanh nghiệp nhỏ. Tuy quá trình ra quyết định không tác động nhiều nhưng hành động của các doanh nghiệp vừa và lớn lại ảnh hưởng đáng kể đến hiệu quả hoạt động của họ.

\section{Bảng 7}

Các trọng số chuẩn hóa trong mô hình khả biến

\begin{tabular}{|l|c|c|}
\hline \multirow{2}{*}{\multicolumn{1}{|c|}{ Mối quan hệ }} & \multicolumn{2}{c|}{ Ước lượng } \\
\cline { 2 - 3 } & Doanh nghiệp nhỏ & Doanh nghiệp vừa và lớn \\
\hline Sự cảm nhận $\rightarrow$ hiệu quả hoạt động & 0,294 & 0,577 \\
\hline Ra quyết định $\rightarrow$ hiệu quả hoạt động & 0,642 & 0,531 \\
\hline Hành động $\rightarrow$ hiệu quả hoạt động & 0,184 & 0,618 \\
\hline
\end{tabular}

Nguồn: Kết quả khảo sát 249 doanh nghiệp (2019)

\section{Kết luận và hàm ý quản trị}

Bài nghiên cứu tiến hành phỏng vấn 249 doanh nghiệp ở TPCT bằng phương pháp chọn mẫu phi xác suất theo kiểu hạn mức nhằm phân tích tác động của sự thích ứng tổ chức đến hiệu quả hoạt động của doanh nghiệp. Kết quả cho thấy, có tồn tại mối quan hệ thuận chiều giữa sự thích ứng và hiệu quả hoạt động của doanh nghiệp. Trong đó, yếu tố liên quan đến sự ra quyết định có tác động mạnh nhất, kế tiếp là sự cảm nhận và hành động của tổ chức. Mặt khác, nghiên cứu cũng cho thấy có sự khác biệt lớn về tác động của sự thích ứng tổ chức đến hiệu quả hoạt động của doanh nghiệp giữa doanh nghiệp quy mô nhỏ, và doanh nghiệp quy mô vừa và lớn.

Kết quả nghiên cứu không chỉ đóng góp trong việc làm giàu minh chứng học thuật về mối quan hệ giữa sự thích ứng tổ chức và hiệu quả hoạt động kinh doanh, mà còn là thông tin khoa học quan trọng giúp các nhà quản trị có định hướng tốt hơn trong việc nâng cao sự thích ứng tổ chức và hiệu quả hoạt động kinh doanh. Cụ thể một số hàm ý quản trị được đề xuất như sau: (1) Các doanh nghiệp nên sử dụng nhiều phương tiện, công nghệ khác nhau để cập nhật tin tức sẽ giúp doanh nghiệp, đặc biệt là các doanh nghiệp nhỏ phát hiện được xu hướng thay đổi trong thị hiếu của khách hàng ở từng vùng miền khác nhau. Doanh nghiệp có thể chú trọng đến công nghệ số trong khâu bán hàng (bán hàng online, ứng dụng quảng cáo, ...) và chú ý đến tính hấp dẫn, khiến khách hàng ngạc nhiên khi nghĩ tới sản phẩm/dịch vụ của mình. Làm được điều này, các doanh nghiệp không những nâng cao khả năng cảm nhận của mình mà còn ảnh hưởng đáng kể đến hiệu quả hoạt động của doanh nghiệp. (2) Các doanh nghiệp cần quan tâm hơn đến việc thường xuyên phân tích các sự kiện, xác định cơ hội và thách thức, thực hiện các kế hoạch đúng thời điểm. Điều này sẽ giúp doanh nghiệp nâng cao hiệu quả cho việc ra quyết định, đồng thời sẽ nâng cao hiệu quả hoạt động kinh doanh cho doanh nghiệp. (3) Nhà quản trị cũng cần 
định hình lại các nguồn lực của mình, cũng như xem xét quy trình kinh doanh nhằm cung cấp những sản phẩm/dịch vụ mới phục vụ khách hàng đúng thời điểm. Doanh nghiệp có thể thiết kế cửa hàng, sản phẩm của mình kết hợp những hoạt động mang lại trải nghiệm độc đáo cho người tiêu dùng. Những hành động đó sẽ tác động đến sự thích ứng (khía cạnh hành động) của doanh nghiệp.

Bài nghiên cứu đã tìm ra được mối quan hệ giữa sự thích ứng và hiệu quả hoạt động của doanh nghiệp. Tuy nhiên, còn nhiều yếu tố trung gian khác có thể ảnh hưởng đến hiệu quả hoạt động thông qua sự thích ứng tổ chức mà bài nghiên cứu chưa đề cập. Đó có thể là ảnh hưởng của sự thích ứng tổ chức đến sự phát triển của tính sáng tạo trong nhân viên/sự gắn kết trong công việc, và hiệu quả hoạt động của doanh nghiệp. Đây là những gợi mở cho những hướng nghiên cứu mới trong tương lai.

\section{Tài liệu tham khảo}

Abolfazl, J. M., \& Mehrdad, K. (2016). The mediating role of organizational agility in the relationship between organizational learning and organizational performance (Case study: The subsidiaries of Isfahan City general tax office). International Business Management, 10(16), 3530-3535.

Alegre, J., \& Sard, M. (2015). When demand drops and prices rise. Tourist packages in the Balearic Islands during the economic crisis. Tourism Management, 46, 375-385. doi:10.1016/j.tourman.2014.07.016

Anas, Y. A. (2016). The effect of organizational agility on organization performance. International Review of Management and Business Research, 5(1), 273-278.

Balaji, M., Elmurugan, V., Sivabalan, G., Ilayaraja, V. S., Prapa, M., \& Mythily, V. (2014). ASCTM approach for enterprise agility. Procedia Engineering, 97, 2222-2231.

Barney, J. B. (1997). Gaining and sustaining competitive advantage. Boston: Addison-Wesley Publishing Company.

Cascio, W. F. (1989). Human resources: Productivity, quality of work life (2nd ed.). New York, NY: McGraw-Hill Education.

Chung, R. G., \& Lo, C. L. (2007). The relationship between leadership behavior and organizational performance in non-profit organizations, using social welfare charity foundations as an example. The Journal of American Academy of Business, Cambridge, 12(1), 83-87.

D’Aveni, R. A. (1994). Hypercompetition: Managing the dynamics of strategic maneuvering. New York, NY: Free Press.

Daft, R. L. (2000). Essentials of organization theory and design. Mason, OH: South-Western College Pub.

Ganguly, A., Nilchiani, R., \& Farr, J. V. (2009). Evaluating agility in corporate enterprises. International Journal of Production Economics, 118(2), 410-423. doi:10.1016/j.ijpe.2008.12.009 
Gerbing, D. W., \& Anderson, J. C. (1988). An updated Paradigm for scale development incorporating unidimensionality and its assessment. Journal of Marketing Research, 25(2), 186-192. doi:10.1177/002224378802500207

Gimeno, J. (2002). The performance effects of unintended purposive multimarket contact. Managerial and Decision Economics, 23(4/5), 209-224. doi:10.1002/mde.1062

Goldman, S. L., Nagel, R. N., \& Preiss, K. (1995). Agile competitors and virtual organizations: Strategies for enriching the customer. New York, NY: Van Nostrand Reinhold.

Griffin, K. (2003). Economic globalization and institutions of global governance. Development and Change 34(5), 789-808. doi:10.1111/j.1467-7660.2003.00329.x

Gunasekaran, A. (1999). Agile manufacturing: a framework for research and development. International Journal of Production Economics, 62, 87-105. doi:10.1016/S09255273(98)00222-9

Hair, J. F., Black, W. C., Babin, B. J., Anderson, R. E., \& Tatham, R. L. (2006). Multivariate data analysis (6th ed.). Upper Saddle River, NJ: Pearson Prentice Hall.

Heffernan, M., \& Flood, P. C. (2000). An exploration of the relationships between the adoption of managerial competencies, organisational characteristics, human resource sophistication and performance in Irish organisations. Journal of European Industrial Training, 24(2/3/4), 128-136. doi:10.1108/03090590010321098.

Highsmith, J. (2004). Agile project management: Creating innovative products. Boston, MA: Addison-Wesley.

Ho, L. A. (2008). What affects organizational performance? The linking of learning and knowledge management. Industrial Management \& Data Systems, 108, 1234-1254.

Hoang, T., \& Chu, N. M. N. (2008). Thống kê úng dụng [Statistics application]. Hanoi, Vietnam: Nhà xuất bản Thống kê.

Hoyle, R. H. (1995). Structural equation modeling: Concepts, issues, and applications. Thousand Oaks, CA: Sage Publications.

Iaccoca Institute. (1991). $21^{\text {st }}$ century manufacturing enterprise strategy: An industry led view. Bethlehem, PA: Lehigh University.

Kaplan, R. S., \& Norton, D. P. (1996). The balanced scorecard. Boston, MA: Harvard Business School Press.

Lagos, R. (2001). A model of TFP. The Review of Economic Studies, 73(4), 983-1007. doi:10.1111/j.1467-937X.2006.00405.x

Maltz, A. C., Shenhar, A. J., \& Reilly, R. R. (2003). Beyond the Balanced Scorecard: Refining the Search for Organizational Success Measures. Long Range Planning, 36(2), 187-204. doi:10.1016/S0024-6301(02)00165-6.

March, J. G. (1991). Exploration and exploitation in organizational learning. Organization Science, 2(1), 71-87. 
Mathiassen, L., \& Pries-Heje, J. (2006). Business agility and diffusion of information technology. European Journal of Information Systems, 15(2), 116-119.

McGaughey, R. E. (1999). Internet technology: Contributing to agility in the twenty-first century. Int, J, Agil, Manag, Syst, 1, 7-13.

Meredith, S., \& Francis, D. (2000). Innovation and strategy journey towards agility: The agile wheel explored. TQM Mag, 12, 137-143.

Najmi, M., Zakuan, N., Rezaei, G., Gholami, H., Shaharou, A. B. M., \& Saman, M. Z. M. (2016). Relationship among culture of excellence, organisational performance and knowledge sharing: Proposed conceptual framework. International Journal of Productivity and Quality Management, 19(4), 446-465. doi:10.1504/IJPQM.2016.10000350

Narasimhan, R., Swink, M., \& Kim, S. W. (2006). Disentangling leanness and agility: An empirical investigation. Journal of Operations Management, 24, 440-457.

Nguyen, D. T., \& Nguyen, T. M. T. (2008). Nghiên cứu khoa học marketing - Úng dụng mô hình cấu trúc tuyến tính SEM [Marketing science research - Applying SEM linear structure model]. Ho Chi Minh, Vietnam: Đại học Quốc gia Thành phố Hồ Chí Minh.

Nguyen, D. K. (2009). Bài giảng: Thưc hành mô hình cấu trúc tuyến tính (SEM) với phần mềm AMOS [Lecture: Practice linear structure model (SEM) with AMOS software]. Trường Đại học Kinh tế Thành phố Hồ Chí Minh, Việt Nam.

Nunnally, J., \& Berstein, I. H. (1994). Pschychometric theory (3rd ed.). New York, NY: McGraw-Hill.

Oosterhout, M. V., Waarts, E., \& Van Hillegersberg, J. (2006). Change factors requiring agility and implications for IT. European Journal of Information Systems, 15(2), 132-145.

Park, Y. (2011). The dynamics of opportunity and threat management in turbulent environments: The role information technologies. (Doctoral dissertation, University of Southern California, Los Angeles, CA). Retrived January 20, 2020, from http://digitallibrary.usc.edu/cdm/ref/collection/p15799coll127/id/621596

Pavlou, P. A., \& Sawy, O. A. E. (2010). The "Third hand": IT-enabled competitive advantage in turbulence through improvisational capabilities. Journal Information Systems Research, 21(3), 443-471.

Raykov, T., \& Widaman, K. T. (1995). Issues in applied structural equation modelling research. Structural Equation Modelling, 2(4), 289-318.

Richards, C.W. (1996). Agile manufacturing: Beyond lean? Production Inventory Management Journal, 37(2), 60-64.

Rigby, C. M., Day, P., \& Forrester, J. B. (2000). Agile supply: Rethinking systems thinking. International Journal Of Agile Management Systems, 2, 534-548.

Sambamurthy, V., Bharadwaj, A., \& Grover, V. (2003). Shaping agility through digital options: Reconceptualizing the role of information technology in contemporary firm. MIS Quarterly, 27(2), 237-263. 
Sanchez, L. M., \& Nagi, R. (2001). A review of agile manufacturing systems. International Journal of Production Research, 39(16), 3561-3600.

Shahrabi, B. (2012). The role of organizational learning and agility in change management in state enterprises: A customer-oriented approach. International Research Journal of Applied and Basic Sciences, 3(12), 2540-2547.

Sharifi, H., \& Zhang, Z. (1999). A methodology for achieving agility in manufacturing organizations: An introduction. International Journal of Production Economics, 62(1/2), 7-22.

Sharifi, H., \& Zhang, Z. (2001). Agile manufacturing in practice - Application of a methodology. International Journal of Operations \& Production Management, 21(5/6), 772-794. doi:10,1108/01443570110390462

Shenhar, A. J., \& Dvir, D. (1996). Long term success dimensions in technology-based organizations. In Handbook of Technology Management. New York, NY: McGraw Hill.

$\mathrm{Su}, \mathrm{G}$. (2011). Exploring requirements of agility for knowledge management. In Wissens management (pp. 371-380).

Sull, D. (2009). How to thrive in turbulent market. Harvard Business Review, 87(2), 78-88.

Tallon, P., \& Pinsonneault, A. (2011). Competing perspectives on the link between strategic information technology alignment and organizational agility: Insights from a mediation model. MIS Quarterly, 35(2), 463-486. doi:10.2307/23044052

Upton, D. M. (1995). What really makes factories flexible? Harvard Business Review, 73(4), 74-84.

Vokurka, R. J., \& Fliedner, G. (1998). The journey toward agility. Industrial Management \& Data Systems, 98, 165-171.

Wadhwa, S., \& Rao, K. S. (2003). Enterprise modeling of supply chains involving multiple entity flows: Role of flexibility in enhancing lead time performance. SIC Journal, 12(1), 5-20.

Wageeh, N. (2016). Organizational agility: The key to improve organizational performance. International Business Research, 9(3), 97-111.

Wageeh, N. (2016). The effect of organizational agility on quality of work life: A study on commercial banks in Egypt. International Journal of Business and Management, 11(6), 270-285.

Zhang, Z., \& Sharifi, H. (2000). A methodology for achieving agility in manufacturing organizations. International Journal of Operations and Production Management, 20, 496-512. 